\title{
Dynamic smoothening and tribological properties of pulsed-DC sputtered DLC based nanocomposite films
}

\author{
K. P. Shaha, Y. T. Pei, C. Q. Chen \& J. Th. M. De Hosson \\ Department of Applied Physics, \\ The Netherlands Materials Innovation Institute (M2i), \\ University of Groningen, The Netherlands
}

\begin{abstract}
Interface roughness and dynamic growth behavior of $\mathrm{TiC} / \mathrm{a}-\mathrm{C}$ nanocomposite films deposited by pulsed-DC magnetron sputtering were studied using atomic force microscopy and scanning electron microscopy. Upon increasing the intensity of concurrent ion impingement by raising the frequency of pulsed-DC sputtering, a transition from dynamic roughening to dynamic smoothening is revealed in the growth behavior of TiC/a-C nanocomposite films. Analyses of surface morphology and growth conditions imply that there is a transition of dominating growth mechanism from geometric shadowing to surface diffusion driven by impact-induced atomistic downhill flow process due to enhanced impingement of Ar+ ions, which occurs upon the change of pulse frequency from $100 \mathrm{kHz}$ to $350 \mathrm{kHz}$. Also ultra-smooth TiC/a-C:H films were successfully grown on initial rough steel substrates $(\mathrm{Sa} \sim 6 \mathrm{~nm})$ by pulsed-DC sputtering at 200 and $350 \mathrm{kHz}$ frequency. These nanocomposite films exhibit superb toughness, wear resistance and ultralow friction.
\end{abstract}

Keywords: dynamic roughening, smoothening, nanocomposite, rough substrates, pulsed-DC sputtering.

\section{Introduction}

Diamond-like-carbon (DLC) is an extensively studied material due to its intriguing physical, mechanical and tribological properties [1]. DLC based nanocomposites incorporating nanosized inclusions are expected to further 
improve the structural and mechanical properties of pure amorphous phases [2]. However, surface smoothness of such films becomes a crucial property for developing frictionless protective coatings. If the sliding surfaces are very rough, a high level of mechanical interlocking between surface asperities leads to high friction and wear losses (especially during the running-in period). During sputter deposition there is interplay between roughening generated by random deposition of atoms, smoothening by surface diffusion and nonlocal effects generated by shadowing. Geometrical shadowing, which results by non-normal incident flux [3], enhances growth front roughness. Without additional lateral relaxation processes, this would inevitably cause dynamic roughening i.e. an increase of surface roughness as a function of deposition time (or film thickness) [4]. A rough interface will induce columnar growth such that the column boundaries (CBs) originate at the groove networks on the growing interface and the hills become the spearheads of the columns. The CBs are potential source of failure under loading and contact sliding. The CBs may act as initiation sites for cracks and preferential cracking paths [2].

The growth mechanisms essentially govern the microstructure and thus influence the mechanical and tribological properties of these films. The substrates used in the industries are inherently rough. Thus it is of practical concern to identify the role of growth mechanisms to optimize the deposition conditions for smooth film topography. Over the last decade considerable attention has been paid to the theoretical and experimental aspects of dynamic roughening of films grown on smooth surface [5-9], whereas little attention has been paid to films grown on rough surfaces where smoothening phenomenon may occur [10-12]. Recently, we have reported the dynamic growth behavior for thick TiC/a-C films grown on smooth surface by pulsed-DC sputtering for different pulse frequencies [13]. Dynamic roughening at low pulse frequency while dynamic smoothening at high pulse frequency was observed.

In this paper, we utilize the fact that pulse frequency has a prominent effect on ion energy and ion flux bombarding at the growing film [14] to investigate the evolution of surface morphology of films grown on rough surfaces during pulsed-DC magnetron sputtering. With increasing the intensity of concurrent ion impingement by raising the frequency of pulsed DC sputtering, a transition from dynamic roughening to dynamic smoothening is revealed. Dynamic smoothening of intentionally grown initial rough $\mathrm{TiC} / \mathrm{a}-\mathrm{C}$ films at higher pulse frequency (350 $\mathrm{kHz}$ ) has been reported. Also mechanical and tribological properties of ultrasmooth TiC/a-C:H films grown on rough steel substrates have been reported.

\section{Experimental}

TiC/a-C nanocomposite films were grown non-reactively on Si using close field unbalanced pulsed-DC magnetron sputtering deposition setup which has been described elsewhere [14]. It consists of two magnetrons, coupled to one Ti and one $\mathrm{Cr}$ target each, powered by DC power supply and two magnetrons, coupled to graphite targets, powered by pulsed-DC power supply. The substrates, located at $80 \mathrm{~mm}$ distant from the targets, were pulsed biased at $-40 \mathrm{~V}(250 \mathrm{kHz}$ 
frequency). No intentional heating was applied. The substrate holder was rotated by $3 \mathrm{rpm}$ to ensure a homogeneous thickness of the top layer. A $200 \mathrm{~nm}$ thick ductile CrTi interlayer of optimized composition and structure was employed to improve the adhesion. Various top layers were deposited for different deposition times and with different pulse frequencies to graphite targets. A rough initial top layer was grown intentionally on the interlayer for $90 \mathrm{~min}$, with $100 \mathrm{kHz}$ frequency and without removing the specimen from the vacuum chamber, keeping all other parameters same, growth was continued for 45 to $180 \mathrm{~min}$ with $350 \mathrm{kHz}$ frequency. The microstructure of the films was characterized with a Philips XL-30S FEG high-resolution scanning electron microscope on fracture cross-sections and a DI NanoScope IIIa atomic force microscope was used to analyze the surface morphology. With the microscope operating in a tapping mode, using a Si tip, the film topography images with a $4 \mu^{2}$ were acquired.

TiC/a-C:H films were deposited on $\varnothing 30 \times 6 \mathrm{~mm}$ discs of hardened M2 tool steel, in an argon/acetylene atmosphere, with the same setup mentioned above but replacing the two graphite targets by two Ti targets. The substrates, located at $80 \mathrm{~mm}$ distant from the targets, were pulsed biased at $-80 \mathrm{~V}(250 \mathrm{kHz}$ frequency). A ductile CrTi interlayer was deposited to improve adhesion. The substrate holder was rotated by $3 \mathrm{rpm}$ to ensure a homogeneous thickness of the top layer. The pulse frequency applied to the Ti targets was varied viz. $200 \mathrm{kHz}$ and $350 \mathrm{kHz}$ to obtain two different TiC/a-C:H films respectively. A calibrated MTS Nano Indenter XP was employed to measure the hardness $(\mathrm{H})$ and Young's modulus (E) of the films with a Berkovich indenter. In order to have reliable statistics for $\mathrm{H}$ and $\mathrm{E}, 20$ indentations in total were configured. The maximum indentation depth for measuring $\mathrm{H}$ and $\mathrm{E}$ was defined at one tenth of the film thickness. Tribo-tests were performed using a CSM tribometer with a ball-ondisc configuration at room temperature, $0.1 \mathrm{~m} / \mathrm{s}$ sliding speed $(\mathrm{V}), 5 \mathrm{~N}$ normal load (L) and 50\% humidity. The wear depth/height of the coated disc and the counterpart ( $\varnothing 6 \mathrm{~mm}$ ball) was in situ monitored with a resolution of $0.02 \mu \mathrm{m}$ by a rotational variable differential transformer (RVDT) sensor during the tribotests, which allowed in situ measurements of the thickness of the transfer films on the surface of the counterpart. A confocal microscope was used to capture 3D images on a wear track for measuring the wear volume.

\section{Results and discussions}

\subsection{Transition from dynamic roughening to smoothening of $\mathrm{TiC} / \mathrm{a}-\mathrm{C}$ nanocomposite films}

As mentioned earlier, understanding the principal factors that govern the surface roughness is not only of fundamental importance for understanding growth mechanisms but also technologically important so as to control the roughness development of growing films. Figure 1 shows the evolution of interface morphology of various $\mathrm{TiC} / \mathrm{a}-\mathrm{C}$ films as a function of growth time. The interlayer exhibits a grainy surface morphology (Fig. 1a) with root mean square (RMS) roughness of $0.29 \pm 0.01 \mathrm{~nm}$. Fig. 1b shows the surface morphology of 
the TiC/a-C nanocomposite film grown (on the smooth interlayer) by pulsed-DC with $100 \mathrm{kHz}$ frequency. It is interesting to note the immense increase in RMS roughness from $0.29 \mathrm{~nm}$ to $5.94 \pm 0.15 \mathrm{~nm}$. This clearly indicates that during the film growth the interface experiences continuous roughening.

a

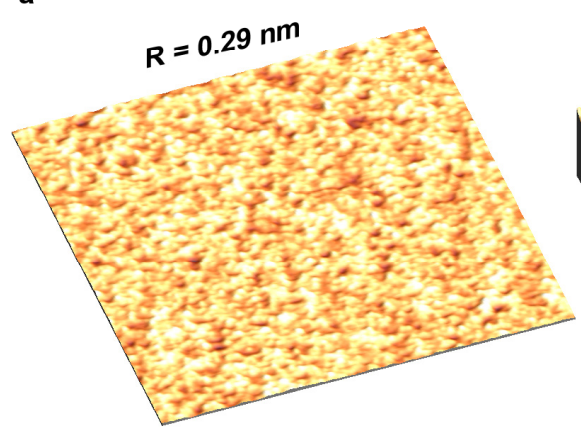

b

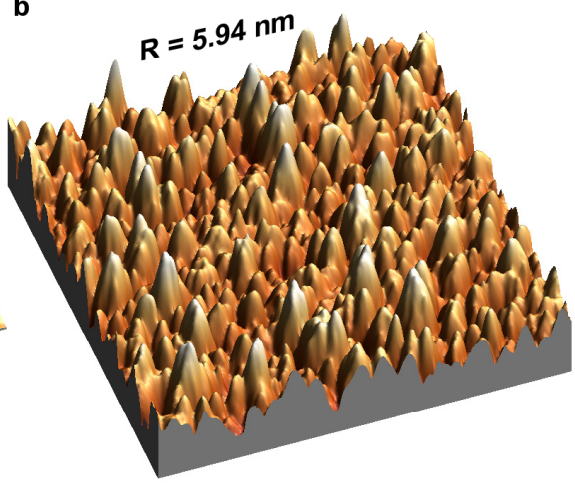

d

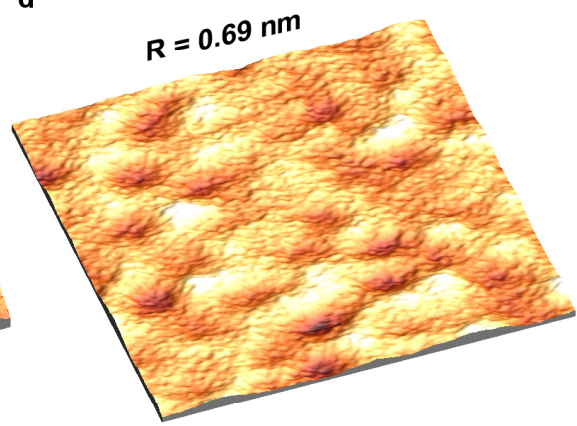

Figure 1: $\quad$ AFM topography images of (a) interlayer; TiC/a-C nanocomposite films grown for (b) 90 min with $100 \mathrm{kHz}$ on interlayer, (c) $45 \mathrm{~min}$ and (d) 180 min with $350 \mathrm{kHz}$ on surface (b). The RMS roughness $(\mathrm{R})$ is noted.

This rough surface ( $\sim 6 \mathrm{~nm}$ RMS roughness) represent the polished substrates used in industry. To check the feasibility of dynamic smoothening effect of pulsed-DC sputtering at high pulse frequency [13] on rough surfaces, intentionally rough $\mathrm{TiC} / \mathrm{a}-\mathrm{C}$ nanocomposite films were first grown with $100 \mathrm{kHz}$ (Fig. 1b) and growth was continued with $350 \mathrm{kHz}$ pulse frequency for different growth time. Fig. 1c and Fig. 1d show the interface morphology of TiC/a-C nanocomposite films grown for $45 \mathrm{~min}$ and $180 \mathrm{~min}$ respectively. The interface roughness decreases from $5.94 \mathrm{~nm}$ to $2.42 \pm 0.04 \mathrm{~nm}$ for $45 \mathrm{~min}$ and subsequently to $0.69 \pm 0.02 \mathrm{~nm}$ for $180 \mathrm{~min}$ film growth with pulsed-DC 350 $\mathrm{kHz}$. The rapid decrease in the surface roughness as a function of growth time, with the onset of growth with $350 \mathrm{kHz}$ frequency is of great interest. This 
indicates the presence of surface diffusion controlled growth. The change in pulse frequency causes a transition of dominating growth mechanism which controls the film growth.

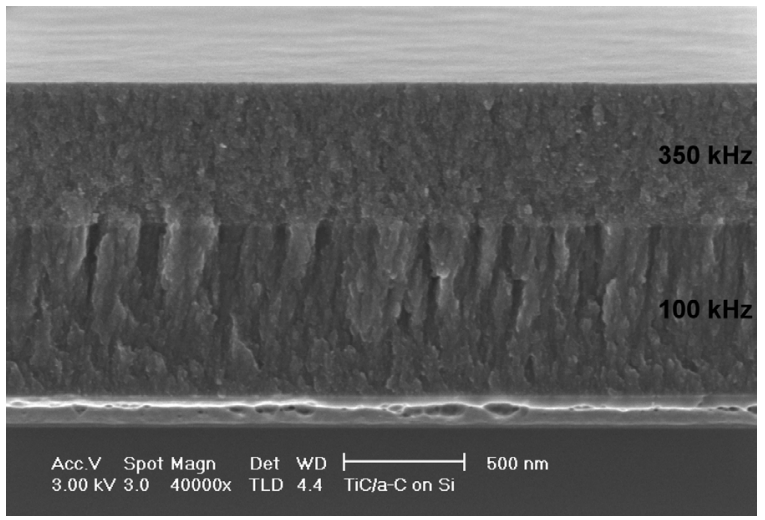

Figure 2: $\quad$ Cross sectional SEM micrograph of TiC/a-C film grown first with $100 \mathrm{kHz}$ for $90 \mathrm{~min}$ followed by $350 \mathrm{kHz}$ for $180 \mathrm{~min}$. An immediate transition of microstructure, from columnar to diffused, was observed with change in pulse frequency from $100 \mathrm{kHz}$ to 350 $\mathrm{kHz}$.

In sputter deposition, the growth dynamics are dominated by nonlocal growth effects. The primary nonlocal effect is geometrical shadowing, where taller surface features block the incoming flux from reaching lower-lying areas of the surface. The shadowing effect is active because, in sputter deposition, the incoming flux has an angular distribution. This allows the taller surface features to grow at the expense of shorter ones, leading to a competition between different surface features for particle flux. This competition, in the absence of lateral relaxation processes leads to interface roughening for films grown at low pulse frequency $(100 \mathrm{kHz})$. However, pulsing the magnetrons at higher pulse frequency results in increased ion energy flux delivered to the growing film [14]. The ion flux and ion energy bombarding at the growing interface plays a crucial role in achieving the smoothening effect.

At $350 \mathrm{kHz}$ frequency a high Ar+ ions and energy flux, among which $80 \%$ of ions carry 50-250 eV, was delivered to the growing film [14]. Also the plasma fills in the whole chamber ensuring continuous impingement of the growing film in a closed-field unbalanced configuration [14]. The Ar+ ions impinge more likely at the surface protrusions or hills rather than at the valleys leading to the development of growth instabilities, which is consistent with a scenario in which shadowing effects operate. As the growth progresses, the hills merge into each other at the bottom of the valleys, which is due to the preferred growth of the valley in the midst of the hills. At the final stage of the growth at $180 \mathrm{~min}$, the surface becomes smoother and the peak-to-valley distance becomes smaller. It is believed that the intensive and continuous impingement with high flux and high 
energy ions cause impact induced downhill flow of adatoms in the presence of top amorphous layer [13] as proposed by Moseler et al. [9]. This surface diffusion competes with the geometrical shadowing and noise induced roughening to evolve surface smoothening. However at $100 \mathrm{kHz}$ the $\mathrm{Ar}+$ ion and energy flux is quite low compared to $350 \mathrm{kHz}$. Also the plasma does not cover the whole chamber [14]. Under these growth conditions the surface diffusion does not provide enough lateral relaxation yielding dynamic roughening.

Fig. 2 shows the fracture cross section of TiC/a-C nanocomposite film grown for 90 min with $100 \mathrm{kHz}$ and subsequently for 180 min with $350 \mathrm{kHz}$ pulse frequency. During the growth with $100 \mathrm{kHz}$ frequency, the microstructure shows a severe columnar structure but at the onset of growth with $350 \mathrm{kHz}$ the microstructure evolves in a dense and non columnar structure. A rough interface induces columnar growth at $100 \mathrm{kHz}$. Thus the interface structure essentially determines the microstructure of the film. And as the growth mechanisms control the interface structure during growth, the restraint of columnar structure can be attributed to change in dominant growth mechanism from geometric shadowing to surface diffusion by impact induced atomistic downhill flow.

\subsection{Ultrasmooth TiC/a-C:H nanocomposite films grown on rough steel substrates}

The surface topography and tribological characterization of TiC/a-C:H nanocomposite films grown by pulsed-DC at higher pulse frequencies on rough steel substrates will be described in the following. Fig. 3 shows the evolution of surface morphology of these films. The RMS roughness of the steel substrate decreases considerably from $1.37 \pm 0.07 \mathrm{~nm}$ to $0.29 \pm 0.01 \mathrm{~nm}$ and $0.19 \pm 0.01$ $\mathrm{nm}$ for films deposited with $200 \mathrm{kHz}$ and $350 \mathrm{kHz}$ respectively. The RMS roughness calculated by AFM (Fig. 3a) for steel substrates is too low as it is scanned over small area $(2 \mu \mathrm{m} \times 2 \mu \mathrm{m})$. So confocal microscopy was used to image the topography of the steel substrates (not shown) and the Sa value (over $290 \mu \mathrm{m} \times 290 \mu \mathrm{m}$ ) was measured as $6 \mathrm{~nm}$ which is typical value for the polished industrial substrates. During the film growth the groove structure (Fig. 3a) breaks
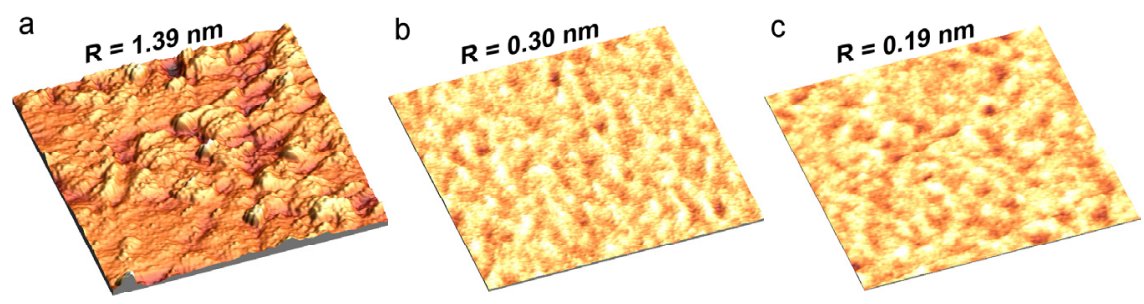

Figure 3: AFM topography images of (a) steel substrate; TiC/a-C:H nanocomposite films grown by pulsed-DC sputtering with pulse frequency: (b) $200 \mathrm{kHz}$ and (c) $350 \mathrm{kHz}$. The RMS roughness (R) is noted. 


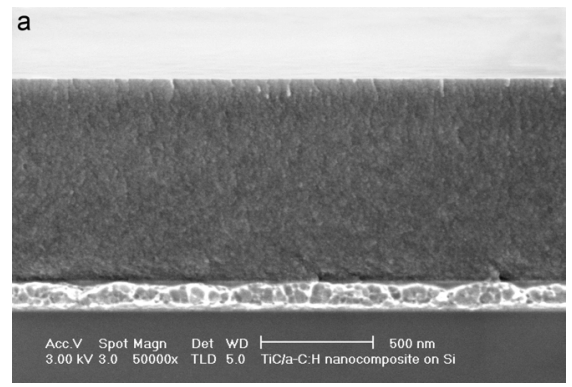

b

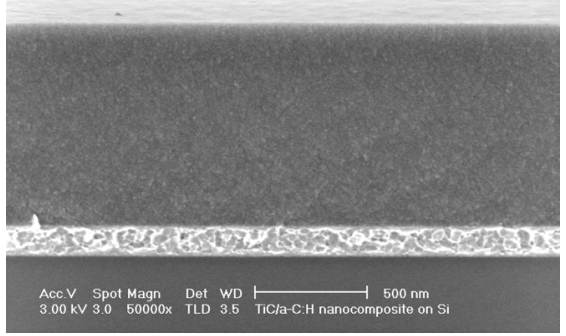

Figure 4: Fracture cross section of TiC/a-C:H films deposited by pulsed-DC reactive sputtering of pulse frequency: (a) $200 \mathrm{kHz}$ and (b) 350 $\mathrm{kHz}$.

down as the hills merge at the bottom of the valleys in the presence of ion impingement and the interface experiences smoothening (Fig. $3 \mathrm{~b}$ and $3 \mathrm{c}$ ). Another contribution to the breaking down of grove network in reactive sputtering is filling in the grooves with carbon adatoms of high mobility [2]. Thus ultra-smooth films can be effectively grown on rough substrates with enhanced ion impingement during film growth by pulsed-DC sputtering at higher pulse frequencies.

As mentioned earlier, the interface structure essentially determines the microstructure of the films. A rough interface induces columnar structure. But the concurrent ion impingement suppresses the column formation and evolves glassy microstructure for films grown by pulsed-DC sputtering at higher pulse frequency as shown in Fig. 4. Such dense films with an amorphous microstructure exhibits a substantial toughening compared to films with columnar structure where nucleation and crack propagation is favoured at the CBs [2].

Fig. 5 shows the tribological characterization of these films. Representative graph of friction coefficient versus running laps for TiC/a-C:H film grown by $\mathrm{p}$ DC $200 \mathrm{kHz}$ is shown in Fig. 5a. It shows not only a low steady-state CoF (0.049), but also a quick drop in the CoF from an initially high value of about 0.18 at the beginning of sliding until the transition point where the steady state is reached. This behavior is attributed to the gradual formation of a transfer film on the counterpart surface during the early stage of a tribo-test, which makes the contact in between two basically similar hydrophobic DLC surfaces that contribute to counterpart surface during the early stage of a tribo-test, which makes the contact in between two basically similar hydrophobic DLC surfaces that contribute to self-lubrication. The interfacial sliding actually takes place between the transfer films on the ball and the surface of the film, rather than sliding between the surfaces of the counterpart and the film. To investigate further the influence of the transfer layer, the wear depth was monitored inset through an RVDT sensor. Segments with a negative slope were observed in the depth vs. lap graph, as marked by the arrows in Fig. 5a. These segments are indicative of the built-up of a transfer layer on the ball surface, rather than a real 

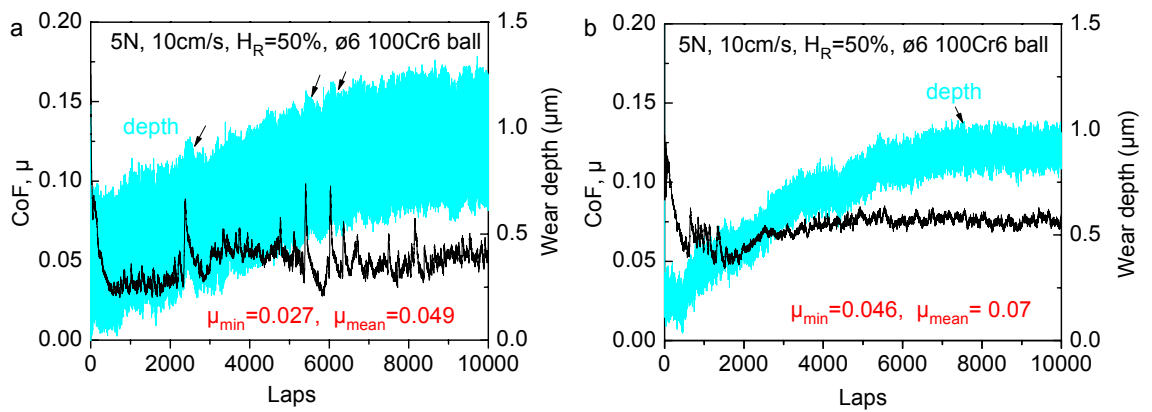

Figure 5: Tribo-test results showing friction coefficient of TiC/a-C:H nanocomposite films on hardened M2 tool steel substrates by pulsed-DC reactive sputtering of frequency (a) $200 \mathrm{kHz}$ and (b) $350 \mathrm{kHz}$.

reduction in the depth of the wear track on the films. Correspondingly, substantial drops in the CoF were detected.

Often peaks were observed in the trace. Their presence can be explained as follows: as the transfer film forms on the counterpart, the CoF cannot decrease further and therefore starts to fluctuate. It can be inferred that since the transfer film covers the ball surface in contact, the wear rate of the film decreases and leads to less wear debris. As a result, the transfer film becomes thinner during sliding until it fully breaks down, leading to a sudden rise of the CoF. Sliding at a higher $\mathrm{CoF}$ can generate more debris from the wear track, which in turn can provide the necessary material for the growth of a new transfer film. Thereafter, a new cycle of the dynamic friction process is repeated. Wear debris collected in front of and beside the wear scar on the ball counterpart were observed under optical microscope (not shown). Similar self-lubrication effect was observed for the film grown by pulsed-DC $350 \mathrm{kHz}$ as shown in Fig. 5b. However, the steady state $\mathrm{CoF}$ was 0.07 . The increase in $\mathrm{CoF}$ is mainly due to the increased hardness of the film grown at $350 \mathrm{kHz}$ compared to $200 \mathrm{kHz}$. Hardness of these films was measured by nanoindentation as 16.1 and $17.1 \mathrm{GPa}$ for 200 and $350 \mathrm{kHz}$ respectively. During sliding (Fig. 5b), there is frequent built-up and rupture of transfer layer formed on the ball counterpart in the presence of hard abrasives. A confocal microscope was used to capture 3D images of each wear track for measuring the wear volume. Eight images of 3D profiles were captured at different positions on a wear track for statistics. A software code was programmed in MatLab to process the 3D images and to calculate the wear rate of the films. The wear rates were calculated as $5 \times 10^{-8}$ and $3 \times 10^{-8} \mathrm{~mm}^{3} /(\mathrm{N}$ lap m) for the films grown with 200 and $350 \mathrm{kHz}$ respectively.

\section{Conclusions}

Dynamic smoothening of DLC based nanocomposite films grown on rough substrates is revealed. It is shown that smoothening of initially rough surfaces with RMS roughness $\sim 6 \mathrm{~nm}$ to RMS roughness $<1 \mathrm{~nm}$ can be effectively 
achieved with pulse-DC sputtering at higher frequency. The ion flux and ion energy bombarding at the growing interface plays crucial role in achieving the smoothening effect and hence column-free microstructure. At low pulse frequencies, the film growth is dominated by geometrical shadowing effect while at high frequency surface diffusion effect due to impact induced downhill flow dominates. Ultrasmooth (RMS roughness $\sim 0.2 \mathrm{~nm}$ ), dense TiC/a-C:H films grown on rough steel substrates exhibit superb toughness, wear resistance and ultralow friction.

\section{Acknowledgements}

This research was carried out under the project number MC7.06246 in the framework of the research programme of the Netherlands Materials Innovation Institute (M2i), the former Netherlands Institute for Metals Research, Delft, the Netherlands. The authors acknowledge financial support from the M2i and the Foundation for Fundamental Research on Matter (FOM-Utrecht), the Netherlands.

\section{References}

[1] J. Robertson, Mater. Sci. Eng., R. 37, 129 (2002).

[2] Y.T. Pei, D. Galvan, J.Th.M. De Hosson, Acta Mater., 53, 4505 (2005).

[3] R.P.U. Karunasiri, R. Bruinsma, J. Rudnick, Phys. Rev. Lett., 62, 788 (1989).

[4] A.L. Barabasi, H.E. Stanley, Fractal Concepts in Surface Growth, (Cambridge Univ. Press, Cambridge, 1995).

[5] H.N. Yang, Y.P. Zhao, G.C. Wang, T.M. Lu, Phys. Rev. Lett., 76, 3774 (1996).

[6] H. You, R.P. Chiarello, H.K. Kim, K.G. Vandervoort, Phys. Rev. Lett., 70, 2900 (1993).

[7] B.Q. Li, I. Kojima, J.M. Zuo, J. App. Phys., 91,4082 (2002).

[8] C. Casiraohi, A.C. Ferrari, R. Ohr, A.J. Flewitt, D.P. Chu, J. Robertson, Phys. Rev. Lett, 91, 226104 (2003).

[9] M. Moseler, P. Gumbsch, C. Casiraghi, A.C. Ferrari, J. Robertson, Science, 309, 1545 (2005).

[10] M.F. Gyure, J.J. Zinck, C. Ratsch, D.D. Vvedensky, Phys. Rev. Lett, 81, 4931 (1998).

[11] B.A. Sperling, R. Abelson, Appl. Phys. Lett., 85, 3456 (2004).

[12] Z.-J. Liu, P.W. Shum, Y.G. Shen, Appl. Phys. Lett., 86, 251908 (2005).

[13] Y.T. Pei, K.P. Shaha, C.Q. Chen, R. van der Hulst, A.A. Turkin, D.I. Vainshtein, J. Th. M. De Hosson, Acta. Mater., (submitted).

[14] Y.T. Pei, C.Q. Chen, K.P. Shaha, J. Th. M. De Hosson, J.W. Bradley, S.A. Voronin, M. Cada, Acta. Mater., 56, 696 (2008). 